This is a postprint version of the following published document:

Martínez-Cisneros, C.S., Gómez-dePedro, S., Puyol, M., García-García, J., Alonso-Chamarro, J. (2012).

Design, fabrication and characterization of micorreactors for high temperatures syntheses.

Chemical Engineering Journal, 211-2012, pp.32-441. DOI: $\underline{10.1016 / j . c e j .2012 .09 .101}$

(C) Elsevier 2012

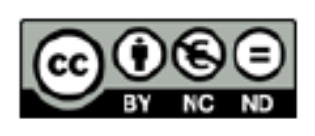

This work is licensed under a Creative Commons Attribution-NonCommercialNoDerivatives 4.0 International License. 


\title{
Design, fabrication and characterization of microreactors for high temperature syntheses
}

\author{
Cynthia S. Martínez-Cisneros ${ }^{a}$, Sara Gómez-de Pedro ${ }^{a}$, Mar Puyol ${ }^{a}$, J. García- \\ García $^{b}$ and Julián Alonso-Chamarro ${ }^{a *}$ \\ ${ }^{a}$ Sensors \& Biosensors Group, Department of Chemistry, Autonomous University of Barcelona, Edifici Cn, 08193 \\ Bellaterra, Catalonia, Spain Fax: 349358 12477; Tel: 349358 12533; E-mail: julian.alonso@uab.es \\ ${ }^{\mathrm{b}}$ Department of Electronic Engineering, Autonomous University of Barcelona, 08193 Bellaterra, Catalonia, Spain.
}

\begin{abstract}
Microfluidic reactors offer many potential advantages in several research and industrial fields such as processes intensification, which includes a better reaction control (kinetics and thermodynamics), a high throughput and a safer operational environment (reduced manipulation of dangerous reagents and low sub-products generation). Nevertheless, scaling-down limitations appear concerning the materials used in the fabrication of microreactors for most of the liquid-phase reactions, since they usually require high temperatures (up to $300^{\circ} \mathrm{C}$ ), solvents and organic reagents. In this work, the development of a set of modular and monolithic microreactors based on the integration of microfluidics and a thermal platform (sensor/high-temperature heater) is proposed to perform high temperature reactions. The reliability and performance of both configurations were evaluated through an exhaustive characterization process regarding their thermal and microfluidic performance. Obtained results make the devices viable for their application in controlled and reproducible synthetic processes occurring at high temperatures such as the synthesis of quantum dots. The proposed microfluidic approach emerge as an engaging tool for processes intensification, since it provides better mass and temperature transfer than conventional methods with a reduction not only of the size and energy consumption, but also of by-products and reagents consumption.
\end{abstract}

Keywords: process intensification, microreactor, high temperature synthetic processes, quantum dots, on-line syntheses, thermal/microfluidic characterization

\section{Introduction}

Conventional chemical syntheses commonly require the use of round-bottomed flasks, beakers, test tubes or even more complex equipments. Additionally, they are usually performed under moderate or high temperatures in organic media, involving the use of oil/sand baths or heat plates. In concrete, the temperature at which a reaction takes place is one of the most important parameters to be considered, since all reactions require a minimal energy to reach the activation threshold that enables to transform the reagents into a desired product. Increasing the temperature in a reaction, not only provides the necessary energy for the reaction to take place, but also favors kinetics [1]. Elimination reactions [2], nucleophilic substitutions [3], thermal cycloadditions [4], intramolecular rearrangements [5] and palladium catalyzed reactions [6] are some of the reactions that demand moderate temperatures. Other reactions involving thermal decompositions require higher temperatures, since in these processes it is necessary to break chemical bonds of reagents [7]. In fact, some nanomaterials syntheses, which nowadays are extensively studied due to their wide range of applications, require temperatures that might reach values up to $300^{\circ} \mathrm{C}$, for example quantum dots synthesis [8-9].

Chemical research is focused on the synthesis of new compounds, their application, and on process intensification. Hence, it is important to develop new equipment or techniques, which drastically improve the whole synthetic process by minimizing energy consumption, size, amount of reagents and by-products and, obviously, the total costs of the process [1, 10-12]. Optimization studies regarding the improvement of reaction yields as well as the minimization of undesired sub-products constitute crucial parameters to take into account. In this sense, the miniaturization concept appears as a promising approach to better control certain reaction parameters. The miniaturization and integration of all the stages associated 
to such procedures would increase the system robustness and would enable its automation, promoting an improved control over some parameters during reactions such as temperature and reaction time. Miniaturized systems offer additional advantages such as the significant reduction of reagents consumption, which is becoming of major importance due to environmental concerns, and safer conditions when reactions involve the manipulation of hazardous reagents.

In recent years, microfluidic systems have been increasingly used for synthetic purposes due to the advantages that they confer and the evolution that they have suffered from simple basic devices to more complex systems [10,13-14]. These systems not only can be directly attached to pre-treatment steps or analytical instruments (such as chromatographs or spectrophotometers) but also can integrate some of them, even monolithically [15-16]. Some studies reveal that the high surface area to volume ratio of microreactors improves mass and heat transfer, giving nearly gradientless conditions, which are desirable for the determination of reaction kinetics [17-18]. This allows carrying out reactions under more precisely controlled conditions than those provided by conventional macroscale reactors $[10,19]$, leading to improved purity and yield of the desired products and minimizing the need of performing collateral reactions and the formation of undesirable by-products [2024]. Moreover, microfluidics represents a better option than conventional methodologies for the synthesis of compounds that require an inert atmosphere, since the environment can be completely controlled in a simple way inside the microfluidic channels. Indeed, the degradation of certain sensitive products can be better avoided inside microreactors [13]. Another relevant advantage is the possibility to automate the whole process through the use of pumps, valves and computer-controlled elements, which increases reproducibility and autonomy, reduces possible risks associated to hazardous reagents and provides realtime information regarding the reaction evolution. As a matter of fact, microfluidic approaches have been successfully applied to the synthesis of numerous metal, metaloxide and semiconductor nanoparticles [25-27].

Miniaturized systems applied as high temperature microreactors are usually implemented using independent modules, where different technological platforms such as microfluidics, thermal modules, detection systems and electronics are integrated. Most of these systems consist on glass, silicon or plastic microfluidic platforms immerged in external oil-baths [2829] or placed over hot plates at high temperatures [30-31]. These modular approaches provide certain advantages that include easy exchangeability/replacement of the platforms in case of malfunction, versatility in case of changing experimental needs and simple design and fabrication. Nevertheless, automatic and more robust devices (monolithic or more compact modular systems) are required for specific applications. Most microreactors found in the bibliography are based on silicon/glass, since these materials provide physical and chemical stability and a well-established fabrication procedure that permits the integration of electronic components such as heaters and sensors (thin-film technology). However, scarce literature related to the development of monolithic microreactors that integrate microfluidics and heating systems can be found in the literature [32-33]. This may respond to the complexity associated to conventional methodologies used for their fabrication. Tiggelaar et al. proposed a high temperature microreactor that included an integrated sensor/actuator system based on the microelectronics technology [30]. The high integration level offered by this microreactor is remarkable but the fabrication process involves high costs, highly skilled staff, special conditions (clean room) and lasting procedures. In order to overcome these issues, alternative technologies based on a simple multilayer approach compatible with the high temperatures associated to most synthetic processes would be desirable. In this sense, the LTCC (Low Temperature Cofired Ceramics) technology provides a way to develop miniaturized monolithic or modular devices that integrate microfluidics, electronics, sensors and actuators (heaters) [34-35]. Their chemical resistivity, once fired in a furnace, makes LTCC devices feasible for chemical applications where organic reagents or corrosive solvents are needed. Moreover, due to the high fusion temperature of materials involved in LTCC technology, up to $850^{\circ} \mathrm{C}$, they are preferable for high temperature applications over other multilayer technologies, such as those based on polymers. Several microreactors based on LTCC materials regarding nanoparticles synthesis [36-38], polymerase chain reaction systems [37], 
enzyme-based determinations [39] and biochemical diagnoses [40], among other applications, can be found in the bibliography.

Herein, we propose the development of highly efficient ceramic microreactors to perform moderate or high temperature reactions. The reliability and performance of modular and monolithic approaches has been evaluated, taking advantage of the properties offered by the LTCC technology, through a detailed hydrodynamic and thermal characterization. To demonstrate the feasibility of the proposed approaches, the modular configuration was applied for the continuous and controlled synthesis of quantum dots, since its synthetic procedure involves temperatures around $300^{\circ} \mathrm{C}$. Nevertheless, since temperature can be precisely controlled as desired, several reactions involving temperature can be carried out in order to obtain nanomaterials or compounds of different nature.

\section{Materials and methods}

DuPont 951 green tapes (thickness at green stage: $254 \mu \mathrm{m}$ ) were used as substrate for the fabrication of modular and monolithic configurations of a set of microreactors involving microfluidic and thermal platforms. The thermal platform consists on an embedded heater screen-printed using gold cofirable conductor paste DuPont 5742 (fired resistivity $<5$ $\mathrm{m} \Omega / \mathrm{sq}$ ). This paste was selected on the basis of its suitability to provide high conductive paths where high power dissipation is a must. Via filling was performed using DuPont 6141 (fired resistivity $<3 \mathrm{~m} \Omega / \mathrm{sq}$ ). Both conductive pastes are perfectively compatible with the whole LTCC fabrication process. As temperature sensor, a commercial class A PT100 (Innovative Sensor Technology, Switzerland) was glued over the device surface by means of epoxy (EPO-TEK® H2OE). A PIC18F4431 microcontroller (Microchip Inc., USA) was used to implement a digital PID (proportional-integral-derivative) control system that maintains temperature at a prefixed value. All the analog/digital electronic components used to implement the control system were carefully selected on the basis of improving the system precision and increasing the signal to noise ratio. The user interface for temperature monitoring during the tests was developed on a personal computer through a virtual instrument specifically designed for this application.

To perform the thermal characterization of the microreactor, an EMC Scanner with an integrated IR Probe (RS321EH, Detectus $A B$ ) was used. The mixing efficiency of the microfluidic structure embedded in the microreactor was evaluated by means of a qualitative visual characterization performed through a stereo microscope Leica S6D equipped with a DFC290 digital camera (Leica Microsystems S.L.U., Spain). For this purpose, two syringe pumps (540060 TSE systems) coupled to $10 \mathrm{ml}$ syringes (Hamilton series GASTIGHT 1000 TLL) connected to the ceramic microfluidic platform with PTFE tubes (i.d. $0.9 \mathrm{~mm}$ ) were applied.

\section{Microsystems fabrication}

The general fabrication process of the LTCC-based microsystems starts with their design using computer assisted design (CAD) software. Since it is a multilayer approach, the design has to be decomposed in layers on the xy-plane, which once overlapped create a desired three-dimensional structure. These CAD designs are transferred to green ceramic tapes by an etching machine (in this case, a laser machine). Then, the screen printing step is performed in those patterned layers that require integrating conductive tracks (such as heaters) or vias to connect conducting tracks at different layers. Once the conductive screen-printing paste is dried, all layers are aligned and laminated by a thermocompression procedure consisting on applying 3000 psi for $60 \mathrm{~s}$ at $90{ }^{\circ} \mathrm{C}$. Finally, the laminated block is sintered in a programmable furnace, where different temperature profiles are applied. The whole process, which takes place in less than one day, is depicted in figure 1 and described in detail elsewhere [41].

As previously mentioned in this work, microreactors for synthetic processes based on modular and monolithic configurations were evaluated and compared. In order to 
characterize the hydrodynamic and thermal performance of both approaches, four devices were designed and fabricated: a modular thermal platform, a modular microfluidic platform, a modular microfluidic platform with exposed channel and a monolithic system with both platforms integrated in the same unit.

Figure $2 \mathrm{~A}$ presents the layers that once overlapped constitute the modular thermal platform. It consists of a nine-layer block with a screen-printed gold-based heater. It is embedded in the middle of the block (fifth layer) to promote a more uniform heat distribution through the $z$-axis. The nine layers that constitute the thermal platform provide it robustness. The heater design was developed on the basis of a radial and uniform distribution of temperature and trying to avoid a highly elevated heat point in the center of the platform. By defining seven laps of a gold-based screen-printed conductor $(0.7 \mathrm{~mm}$ width, $8 \mu \mathrm{m}$ height), a $13 \Omega$ heater was obtained. External pads were defined on the top layer of the thermal platform to connect the embedded heater to an external electronic control set-up, since the high working temperatures associated to the microreactor avoids the surface integration of electronic devices involved by the control system. For temperature sensing and establishing the PID control feedback, a class A PT100 sensor was placed over the thermal platform trying to obtain the optimum xy-alignment with the microreactor zone, which was embedded in the modular microfluidic platform later attached.

The layers that once overlapped constitute the modular microfluidic platform are shown in figure 2B. The microfluidic structure includes two inlets for reagents and one outlet through which the product of the reaction is collected. The complete microfluidic platform consists of eight stacked layers, where the microreactor is embedded. The two inlets allow introducing the reagents into the system through two simple channels around the microreactor before they meet each other downstream. This structure acts as a pretreatment step for preheating reagents before mixing. Channels converge in a Y-shape point downstream before getting into the microreactor (Z-shape mixer). A bi-dimensional micromixer (200 $\mu \mathrm{m}$ width, $200 \mu \mathrm{m}$ height) was preferred for this application. It introduces the chaotic mixing profile required for faster and more homogeneous mixing, avoiding thermal fluctuations on the liquid, which could be produced if three dimensional mixers are used. The circular configuration of the micromixer was selected in accordance to the expected radial thermal distribution regarding the heater embedded in the thermal platform. Therefore, a proper alignment between both platforms, when mechanically attached, would provide a more uniform heat distribution on the xy plane of the microchannel. Using this approach, reagents are exposed to specific, uniform and controlled temperature levels provided by the thermal platform during their flow through.

To perform a qualitative visual characterization of the fluidics/hydrodynamics of the microreactor, a new modular microfluidic platform, identically to that previously presented, was constructed; but leaving half of the microfluidic structure open to air. In this case, once sinterized, the exposed section of the device was covered with a polymeric foil, permitting its inner visualization but ensuring its hermetic sealing (see figure $1 \mathrm{C}$ ).

Finally, to estimate the advantages provided by a monolithic device in terms of heat distribution, taking advantage of the properties provided by the LTCC technology, a microsystem including the microfluidic and thermal platforms in the same device was fabricated. The monolithic design was based on those previously exposed for modular platforms.

\section{Electronic set-up for temperature control}

Since temperature level and distribution are crucial parameters to be considered for several reactions to properly take place, a dedicated temperature controller with a digital PID topology was designed and implemented on a PIC18F4431 microcontroller, using a personal computer for monitoring purposes. The electronic circuit for temperature control was separately fabricated to avoid that the high temperatures at which the microreactor operates would produce any damage to it. A block diagram of the electronics implemented in this work is shown in Figure 3. 
The analog stage of the electronic circuit was designed for conditioning the signal provided by the PT100 sensor, keeping intensity constant through it for avoiding its self heating, and amplifying the control signal generated by the PID controller (a pulse width modulated signal, PWM) before being applied to the embedded heater (actuator). The feedback signal obtained from the PT100 sensor was used to estimate the error and to correct it using specific differential equations programmed in the digital PID control system implemented in the microcontroller code. The control signal produced by the PID was amplified and applied to the gold-based heater embedded into the thermal platform. The thermal platform was configured to work at a wide temperature range $\left(180^{\circ} \mathrm{C}-300^{\circ} \mathrm{C}\right)$ in order to fulfill needs associated to specific synthetic process under evaluation. Nevertheless, such ceramic microreactors could be applied for any reaction performed at higher temperatures, where other materials cannot operate, since its fusion temperature is up to $850^{\circ} \mathrm{C}$.

\section{Results and discussion}

To demonstrate the microreactor performance when applied to synthetic processes, it was thermally and hydraulically characterized. In this sense, its mixing capabilities and thermal distribution were characterized.

\subsection{Fluidic characterization}

To demonstrate that the microfluidic structure designed and embedded into the microsystem was able to provide an efficient mixture of reagents, a qualitative visual characterization of its fluidics/hydrodynamics was performed. For this study, the modular microfluidic device with half of its structure exposed was used. Using this approach, it was possible to examine its interior by means of a stereo microscope. To probe the reagents mixture inside the Z-shape bi-dimensional channel proposed as mixer, different tests using dyes (phenol red and methylene blue, both $1 \mathrm{mM}$ and propelled by syringe pumps at 60 $\mu \mathrm{L} / \mathrm{min}$ ) at room temperature were performed inside the microreactor. For this purpose, four different points of the microchannel were selected the visual exploration (see frames 1, 2, 3 and 4 presented in figure 4 ).

Sequence depicted by frames $1,2,3$ and 4 in figure $4 \mathrm{C}$ allows visualizing the mixing process while reagents flow through the microreactor. When dyes go through the region enclosed by frame 1, both dyes can be clearly differentiated, showing a near ideal laminar flow profile. On the contrary, by the time that reagents reach the zone enclosed by frame 3 , an almost homogeneous coloration can be observed, which results complete when the liquid reaches the zone framed in 4 . Thus, it can be stated that a good mixture of both dyes is achieved before the end of the first circular turn, which makes clear the efficiency provided by the micromixer embedded into the LTCC microreactor.

\subsection{Thermal characterization}

Once the PID control parameters were optimized, a highly stable temperature profile was obtained during all the studies performed in this work. The system was predefined to take about ten minutes to stabilize before reaching the steady state at the desired temperature. A slow response with soft temperature transitions was preferred in order to avoid abrupt thermal changes that could stress/brake the thermal platform. A small peak overshoot of about $8^{\circ} \mathrm{C}$ was observed at the beginning of the control system operation. Signal variations in the order of $\pm 0.5^{\circ} \mathrm{C}$ were observed on the steady state of the system response.

As previously mentioned in this work, modular and monolithic configurations were developed and tested in order to obtain microreactors with an efficient configuration. A modular approach (microfluidic and thermal platforms separately fabricated and later 
attached) increases the system reliability. If a thermal unit or a microfluidic platform with different characteristics is required, it can be replaced without requiring rebuilding the complete device. Moreover, if any of the platforms presents operational problems it can be replaced without affecting the other elements. On the other hand, a monolithic configuration may be preferred for those applications that require dedicated devices with a higher integration level, robustness and the minimum of movable elements. All the evaluation procedures regarding modular and monolithic configurations were performed at $270^{\circ} \mathrm{C}$ and using the EMC scanner with an IR Probe for obtaining the corresponding isothermal maps.

\subsubsection{Modular system}

As a first approach, the isothermal map regarding both sides of the modular thermal platform was obtained. Since maps at both sides were highly similar, only one of them is presented in figure 5A. Figure 5B shows the isothermal map coupled to a picture of the experimental set-up used during this experiment. As seen in this figure, a radial and uniform heat distribution in the xy plane is obtained over the thermal platform. Small deformations at the limits of the device are assumed to be caused by the presence of the metallic connectors used to energize the embedded heater. Regarding the z-axis thermal distribution, punctual measurements at the top and the bottom of the device were carried out at the zone where the temperature sensor was placed. As expected, both measurements corresponded to the predefined set point: $270^{\circ} \mathrm{C}$. This concordance can be associated to an efficient thermal distribution over the z-axis.

Once the modular thermal platform was evaluated, the modular microfluidic platform was mechanically coupled taking care on obtaining the highest surface contact regarding an optimum heat transfer between them. Heat transfer between platforms was favored by the thermal conductivity presented by LTCC materials $(3 \mathrm{~W} / \mathrm{mK})$. Figure $5 \mathrm{C}$ presents a picture of the experimental set-up that includes the isothermal map obtained on the microfluidic platform once coupled to the thermal one. As seen in this figure, a radial distribution over the region corresponding to the placement of the microchannels (see red lines in figure $5 \mathrm{C}$ ) inside the microfluidic platform was obtained, assuring a xy uniform thermal distribution. To determine the thermal gradient in the z-axis, punctual measurements were performed on both sides of the microsystem (thermal and microfluidics). Temperature was found to be $270^{\circ} \mathrm{C}$ and $255^{\circ} \mathrm{C}$ on the thermal and microfluidic platform, respectively. The difference observed might be caused not only by the thermal transfer among the different number of ceramic layers that compose both platforms, but also by the presence of the air gap at the coupling region. The effect of the air gap can be minimized by the use of a thermal conductive paste that favors heat transfer among the surfaces in contact. To estimate the temperature inside the embedded microfluidic channel, a scheme regarding layers distribution and temperature measurements was performed (see figure 5D). According to this diagram and considering the material thermal transfer coefficient, a temperature of $268.2^{\circ} \mathrm{C}$ can be assumed inside the microfluidic channel, which is pretty close to the desired temperature predefined as set point $\left(270^{\circ} \mathrm{C}\right)$. In this sense, a temperature gradient of about $1.8^{\circ} \mathrm{C} /$ layer was estimated.

\subsubsection{Monolithic system}

To evaluate the advantages provided by a monolithic device in terms of heat distribution, a microsystem with a monolithic configuration including the microfluidic and thermal platform in the same device was developed and characterized in a similar way than the modular approach was.

Figure 6 shows the isothermal map obtained on both sides of the monolithic microsystem (top and bottom). As seen in this figure, a radial and uniform distribution pretty similar to that obtained using the modular approach was obtained on both sides of the monolithic microsystem. In this case, punctual temperature measurements were also performed in the regions corresponding to the sensor position. Temperature values of $270^{\circ} \mathrm{C}$ and $260^{\circ} \mathrm{C}$ 
were obtained at the bottom and the top of the microsystem, respectively. Following the same procedure as previously with the modular configuration, the z-axis gradient was estimated. In this case, a temperature gradient of $1.2^{\circ} \mathrm{C}$ per layer was obtained. This improvement regarding results obtained with the modular configuration may respond to the elimination of the air gap between platforms. This favors heat transfer among platforms since no additional interface is introduced. In this case, the temperature inside the microchannel might be estimated to be $268.8^{\circ} \mathrm{C}$.

\subsection{Proof of concept: microreactor application in the synthesis of quantum dots}

To validate the performance of the proposed microreactors in high temperature synthetic processes, the synthesis of CdSe quantum dots was performed, since its formation depends strongly on the temperature applied and its fluctuation [42-43]. The reaction consisted on mixing two precursor solutions, one containing selenium and other with cadmium in a 1:1 molar ratio. The solution mixtures consisted of other chemical reagents for conferring stabilization to the nanocrystals formed [44-45]. The selenium precursor was prepared by mixing $197.4 \mathrm{~g}$ (2.5 mmols) of selenium powder (99.5\%, Aldrich) with $5 \mathrm{~mL}$ of trioctylphosphine (TOP, $10.1 \mathrm{mmols}, 90 \%$, Aldrich) and $10 \mathrm{~mL}$ of oleylamine (OLA, 21.25 mmols, $70 \%$, Aldrich) at room temperature. The cadmium solution consisted of $321.25 \mathrm{mg}$ of cadmium oxide (CdO, 2.5 mmols, $99.99 \%$, Aldrich), $4 \mathrm{~mL}$ of oleic acid (OA, $12.6 \mathrm{mmols}$, Ph Eur, Fluka), $3 \mathrm{~g}$ of tryoctylphosphine oxide (TOPO, $7.8 \mathrm{mmols}, 99 \%$, Aldrich), $5 \mathrm{~mL}$ of OLA (15.2 mmols) and $3 \mathrm{~mL}$ of 1-octadecene (ODE, 90\%, Aldrich). Once precursors were freshly prepared, they were introduced in two syringe pumps, which propelled the solutions to the microreactor at a continuous flow rate of $60 \mu \mathrm{L} / \mathrm{min}$. As commented before, the temperature of the heating platform was fixed at $270{ }^{\circ} \mathrm{C}$. Since CdSe nanocrystals present colored solutions and fluorescent properties, the absorbance and fluorescence properties of the nanoparticles obtained were characterized. Figure 7A shows the spectra recorded and an image of the solution obtained under visible (absorbance spectrum, Shimadzu UV310PC UV-Vis-NIR, Kyoto, Japan) and UV light (fluorescence spectrum, Fluorolog ${ }^{\circledR}$ Modular Spectrofluorometer, Horiba Jobin Yvon).

As can be noticed, the spectra are well defined, indicating the formation of uniform nanocrystals [42], whose properties correspond to those expected according to the working temperature predefined in the microreactor configuration. The shape and size of the nanocrystals were also characterized by transmission electron microscopy (TEM) (JEOL 2011, Tokyo, Japan), obtaining, as expected, uniform quantum dots with an average size of $4.4 \pm 0.4 \mathrm{~nm}$ (Figure 7B). Moreover, no incompatibility between the material and the organic solvents used was observed, which makes clear the proper selection of the ceramic material for reactions involving organic solvents at moderate or high temperatures. According to these results, the correct function of the LTCC-based microreactor can be assumed and extrapolated to the synthesis of other nanoparticles/nanomaterials or other reactions where high and accurate temperatures are required.

\section{Conclusions}

To summarize, ceramic microreactors have been proposed for their application in reactions involving solvents and organic reagents at moderate or high temperatures. The approaches proposed take advantage of microfluidics, since when compared with conventional methods the waste of reagents and the generation of residues is drastically reduced. Moreover, the possible automation of the whole process not only confers a safer operational environment, but also introduces a stricter control over the whole chemical process (higher reproducibility, repeatability, etc.). The ceramic substrate material confers physical and chemical resistivity to the microreactor, being feasible to carry out reactions involving organic solvents. Additionally, the high working temperatures affordable with these materials make possible to perform synthesis at high temperatures, where other microfabrication materials such as polymers cannot operate. On the other hand, the 
fabrication technology of the ceramic microsystems is rapid and does not require special facilities such as clean rooms or specialized staff, which leads to low cost devices.

Two different approaches have been presented, one based on a modular configuration and other on a monolithic approach. The modular proposal confers the possibility of exchanging/replacing one of the modules in case of malfunction or different experimental requirements. On the other hand, it has been observed that the monolithic approach confers more confident results, since the absence of an air gap between platforms enables a better thermal transfer and, therefore, a more uniform temperature distribution along the z-axis (lower temperature gradient). This approach should be used in those cases where the synthetic procedure is well established (dedicated devices) and the temperature plays a crucial parameter. After a hydrodynamic and thermal characterization, both approaches were defined as a good option for being applied to the chemical syntheses with specific temperature requirements. Hydrodynamic characterization with dyes has showed up the good grade of mixture obtained by the bidimenional micromixer introduced. Likewise, the thermal characterization applied to both approaches (modular and monolithic) demonstrated a radial and uniform distribution not only over its surface but also in its inner. As a proof of concept, the modular configuration was applied to the synthesis of nanocrystals, since its formation requires a strict control of several conditions, including temperature, obtaining comparable results to those obtained in the literature, which entails the perfect operation of the microsystem.

\section{Acknowledgements}

This work has been supported by the Spanish Ministry of Science and Innovation (MICINN) through projects CTQ2009-12128 and the Consolider Ingenio 2010 project CSD2006 -12 and Catalonia Government through SGR 2009 -0323.

\section{References}

[1] M. Smith, J. March, March's Advanced Organic Chemistry, Reactions, Mechanisms, and Structure, $6^{\text {th }}$ ed., Wiley-Interscience, Hoboken, 2007.

[2] D. M. Crumpton, K. I. Goldberg, Five-coordinate intermediates in carbon-carbon reductive elimination reactions from Pt(IV), J. Am. Chem. Soc. 122 (5) (2000) 962-963.

[3] I. Roth, S. Spange, Kinetic Studies on the Nucleophilic Aromatic Substitution of Fluoronitrobenzene Derivatives with Polyvinylamine in Water Mediated by 2,6-O-Dimethyl- $\beta$-cyclodextrin, Macromolecules 38 (2005) 10034-10041.

[4] S. Abele, S. Höck, G. Schmidt, J.-A. Funel, R. Marti, High-temperature Diels-Alder reactions: Transfer from batch to continuous mode, Org. Process Res. Dev. DOI: 10.1021/op200320w.

[5] J. A. Rincón, M. Barberis, M. González-Esguevillas, M. D. Johnson, J. K. Niemeier, W. M. Sun, Safe, convenient ortho-Claisen thermal rearrangement using a flow reactor, Org. Process Res. Dev. 15 (6) (2011) 1428-1432.

[6] T. Laird, Book Review of Catalysis of Organic Reactions, Org. Process Res. Dev. 13 (6) (2009) 14321432.

[7] M. Damm, T. N. Glasnov, C. O, Kappe, Translating High-Temperature Microwave Chemistry to Scalable Continuous Flow Processes, Org. Process Res. Dev. 14 (1) ( 2010) 215-224.

[8] J. Park, J. Joo, S. G. Kwon, Y. Jang, T. Hyeon, Synthesis of monodisperse spherical nanocrystals, Angew. Chem., Int. Ed., 46 (2007) 4630-4660.

[9] M. Salavati-Niasari , F. Davar, Z. Fereshteh, Synthesis and characterization of ZnO nanocrystals from thermolysis of new precursor, Chem. Eng. J., 146 (3) (2009) 498-502.

[10] W.-Y. Lin, Y. Wang, S. Wang, H.-R.Tseng, Integrated microfluidic reactors, Nano Today 4 (2009), 470-481.

[11] D. Webb, F. Jamison, Continuous flow multi-step organic synthesis, Chem. Sci. 1 (2010) 675-680.

[12] C. Wiles, P. Watts, Continuous flow reactors, a tool for the modern synthetic chemist, Eur. J. Org. Chem. (2008) 1655-1671.

[13] C. Wiles, P. Watts, Continuous flow reactors: a perspective, Green Chem. 14 (2012) 38-54.

[14] R. L. Hartman, K. F. Jensen, Microchemical systems for continuous-flow synthesis, Lab Chip 9 (2009) 2495-2507.

[15] E. M. Chan, A. P. Alivisatos, R. A. Mathies, High-temperature microfluidic synthesis of CdSe nanocrystals in nanoliter droplets, J. Am. Chem. Soc.127 (2005) 13854. 
[16] J. Kobayashi, Y. Mori, K. Okamoto, R. Akiyama, M. Ueno, T. Kitamori, et al., A microfluidic device for conducting gas-liquid-solid hydrogenation reactions, Science 304 (2004) 1305.

[17] K. Wang, Y.C. Lu, Y. Xia, H.W. Shao, G.S. Luo, Kinetics research on fast exothermic reaction between cyclohexanecarboxylic acid and oleum in microreactor, Chem. Eng. J., 169 (1-3) (2011) 290-298. [18] G. Arzamendi, I. Uriz, P.M. Diéguez, O.H. Laguna, W.Y. Hernández, A. Álvarez, M.A. Centeno, J.A. Odriozola, M. Montes, L.M. Gandía, Selective CO removal over Au/CeFe and CeCu catalysts in microreactors studied through kinetic analysis and CFD simulations, Chem. Eng. J., 167 (2-3) (2011) 588596.

[19] J.-I. Yoshida, A. Nagaki, T. Yamada, Flash chemistry: Fast chemical synthesis by using microreactors, Chem. Eur. J. 14 (2008) 7450-7459.

[20] H. Song, D. L. Chen, R. F. Ismagilov, Reactions in droplets in microfluidic channels, Angew. Chem. Int. Ed. 45 (2006) 7336-7356.

[21] X. Zhang, E.S. M. Lai, R. Martin-Aranda, K. L. Yeung, An investigation of Knoevenagel condensation reaction in microreactors using a new zeolite catalyst, Appl. Catal., A, 261 (1) (2004) 109-118.

[22] J. Ma, M. Zhang, L. Lu, X. Yin, J. Chen, Z. Jiang, Intensifying esterification reaction between lactic acid and ethanol by pervaporation dehydration using chitosan-TEOS hybrid membranes, Chem. Eng. J., 155 (3) (2009) 800-809.

[23] W. Ferstl, M. Schwarzer, S. Loebbecke, E. Fritz-Langhals, J. Stohrer, Process optimization of a catalyzed bleach oxidation for the production of functionalized aldehydes using microreaction technology, Chem. Eng. J., 135 (S1) (2008) S292-S297.

[24] Y. S.S. Wan, K. L. Yeung, A. Gavriilidis, TS-1oxidation of aniline to azoxybenzene in amicrostructuredreactor, Appl. Catal., A, 281 (1-2) (2005) 285-293.

[25] Y. Song, J. Hormes, C.S.S.R. Kumar, Microfluidic synthesis of nanomaterials, Small 4 (2008) 698-711. [26] J. Kim, Z. Li, I. Park, Direct synthesis and integration of functional nanostructures in microfluidic devices, Lab on a Chip 11 (2011) 1946-1951.

[27] S. Gómez-de Pedro, C. S. Martínez-Cisneros, M. Puyol, J. Alonso-Chamarro, Microreactor with integrated temperature control for the synthesis of CdSe nanocrystals, Lab Chip 12 (2012) 1979-1986.

[28] A.M. Nightingale, S.H. Krishnadasan, D. Berhanu, X. Niu, C. Drury, R. Mclntyre, E. Valsami-Jones, J.C. deMello, A stable droplet reactor for high temperature nanocrystal synthesis, Lab Chip 11 (2011) 1221-1227.

[29] H. Yang, W. Luan, S. Tu and Z. M. Wang, High temperature synthesis of CdSe nanocrystals in a serpentine microchannel: wide size tenability achieved under a short residence time, Cryst. Growth Des 9 (2009) 1569-1574

[30] R. M. Tiggelaar, P. van Male, J. W. Berenschot, J. G. E. Gardeniers, R. E. Oosterbroek, M. de Croon, J. C. Schouten, A. van den Berg and M. C. Elwenspoek, Fabrication of a high-temperature microreactor with integrated heater and sensor patterns on an ultrathin silicon membrane, Sens. Actuators A 119 (2005) 196-205.

[31] B. K. H Yen,. A. Gunther, M. A. Schmidt, K. F. Jensen and M. G. Bawendi, A microfabricated gasliquid segmented flow reactor for high-temperature synthesis: the case of CdSe quantum dots, Angew. Chem, Int. Ed. 117 (2005) 5583-5587.

[32] E. M. Chan, A. P. Alivisatos and R. A. Mathies, High-temperature microfluidic synthesis of CdSe nanocrystals in nanoliter droplets, J. Am. Chem. Soc. 127 (2005) 13854-13861.

[33] S.-T. Tu, X. Yu, W. Luan, H. Löwe, Development of micro chemical, biological and thermal systems in China: A review, Chem. Eng. J., 163 (3) (2010) 165-179.

[34] C.S. Martinez-Cisneros, N. Ibanez-Garcia, F. Valdes and J. Alonso, LTCC microflow analyzers with monolithic integration of thermal control, Sens. Actuator A-Phys. 138 (2007) 63-70.

[35] K. Malecha, D.G. Pijanowska, L.J. Golonka and W. Torbicz, LTCC microreactor for urea determination in biological fluids, Sens. Actuator B-Chem. 141 (2009) 301-308.

[36] S. Gomez-dePedro, M. Puyol, D. Izquierdo, I. Salinas, J.M. delaFuente, J. Alonso-Chamrro, A ceramic microreactor for the synthesis of water soluble CdS and CdS/ZnS nanocrystals with on-line optical characterization, Nanoscale 4 (2012) 1328-1335.

[37] P. Bembnowicz, M. Malodobra, W. Kubicki, P. Szczepanska, A. Gorecka-Drzazga, J. Dziuban A. Jonkisz, A. Karpiewska, T. Dobosz, L. Golonka, Preliminary studies on LTCC based PCR microreactor, Sensors and Actuators B 150 (2010) 715-721.

[38] S. Gómez-de Pedro, M. Puyol, J. Alonso-Chamarro, Continuous flow synthesis of nanoparticles using ceramic microfluidic devices, Nanotechnology 21 (2010) 415603.

[39] M. Baeza, C. Lopez, J. Alonso, J. Lopez-Santin, G. Alvaro, Ceramic Microsystem incorporating a microreactor with immobilized biocatalyst for enzymatic spectrophotometric assays, Anal. Chem. 82 (2010) 1006-1011.

[40] D.J. Sadler, R. Changrani, P. Roberts, C.F. Chou, F. Zenhausern, Thermal management of BioMEMS: Temperature control ceramic-based PCR and DNA detection devices, IEEE Transactions on Components and Packaging Technologies 26 (2003) 309-316.

[41] N. Ibanez-Garcia, C.S. Martinez-Cisneros, F. Valdes and J. Alonso, Green-tape ceramics. New technological approach for integrating electronics and fluidics in microsystems, TRAC-TRENDS in Analytical Chemistry 27 (2008) 24-33.

[42] L. Qu and X. Peng, Control of Photoluminescence Properties of CdSe Nanocrystals in Growth, J. Am. Chem. Soc., 124 (2002) 2049-2055 
[43] J. Park, J. Joo, S. G. Kwon, Y. Jang, and T. Hyeon, Synthesis of monodisperse spherical nanocrystals, Angew. Chem., Int. Ed., 46 (2007) 4630-4660.

[44] H. Yang, W. Luan, S. Tu and Z. M. Wang, High-Temperature Synthesis of CdSe Nanocrystals in a Serpentine Microchannel: Wide Size Tunability Achieved under a Short Residence Time, Cryst. Growth Des., 9 (2009) 1569-1574.

[45] W. Luan, H. Yang, S. Tu and Z. Wang, Open-to-air synthesis of monodisperse CdSe nanocrystals via microfluidic reaction and its kinetics, Nanotechnol. 18 (2007) 175603. 
Figure 1. Fabrication procedure of the LTCC microsystem.

Figure 2. Layer by layer design of both platforms integrating the LTCC microreactor. A: Thermal platform; a: top layer (x4); b: screen-printed heater $(x 1)$; c: bottom layer $(x 4)$. B: Picture of the thermal platform fabricated. C: Microfluidic platform; layers a and $\mathbf{f}$ were fabricated by duplicate; the microfluidic structure was embedded at layer e. D: Picture of the microfluidic platform, which includes an exposed section regarding the micromixer.

Figure 3. Block diagram of the digital/analog electronics for an accurate control of temperature inside the microreactor.

Figure 4. Fluidic/hydrodynamic qualitative visual characterization of the microfluidic system. A: Picture of the open to air microfluidic platform covered with a polymeric foil. B: Three-dimensional inner view of the microfluidic platform. C: Photographs corresponding to frames at different points of the micromixer.

Figure 5. Thermal characterization of the microreactor based in a modular configuration. A: Isothermal map obtained on the surface of the modular thermal platform. B: Isothermal map of the modular thermal platform overlapped to a picture of the experimental set-up. C: Isothermal map obtained on the surface of the modular microfluidic platform coupled to the thermal one. D: Schematic diagram of the temperature analysis developed to estimate temperature inside the microfluidic channel. In B and $C$, punctual temperature measurements in the zone of the sensor are indicated.

Figure 6. Isothermal maps obtained on the bottom (A) and the top (B) of the monolithic microsystem. Punctual temperature measurements in the zone of the sensor are indicated (set point of the control system: $270^{\circ} \mathrm{C}$ ).

Figure 7. A: Absorbance $(584 \mathrm{~nm})$ and emission fluorescence $(596 \mathrm{~nm})$ spectra of the $\mathrm{CdSe}$ quantum dots synthesized in the modular microreactor. Images of the solutions of the obtained nanoparticles under visible and UV light can be also observed. B: TEM image from the quantum dots synthesized. 


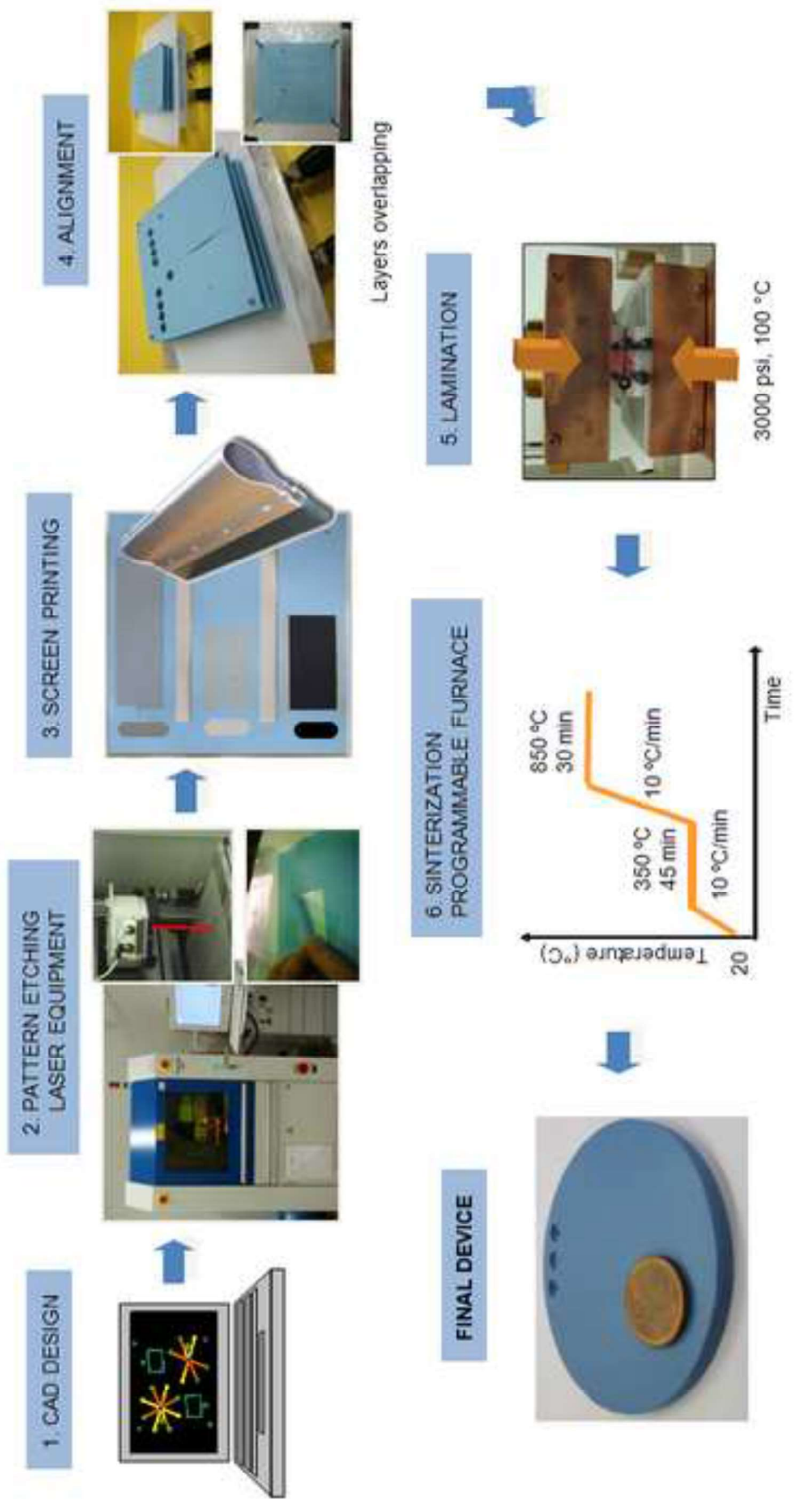




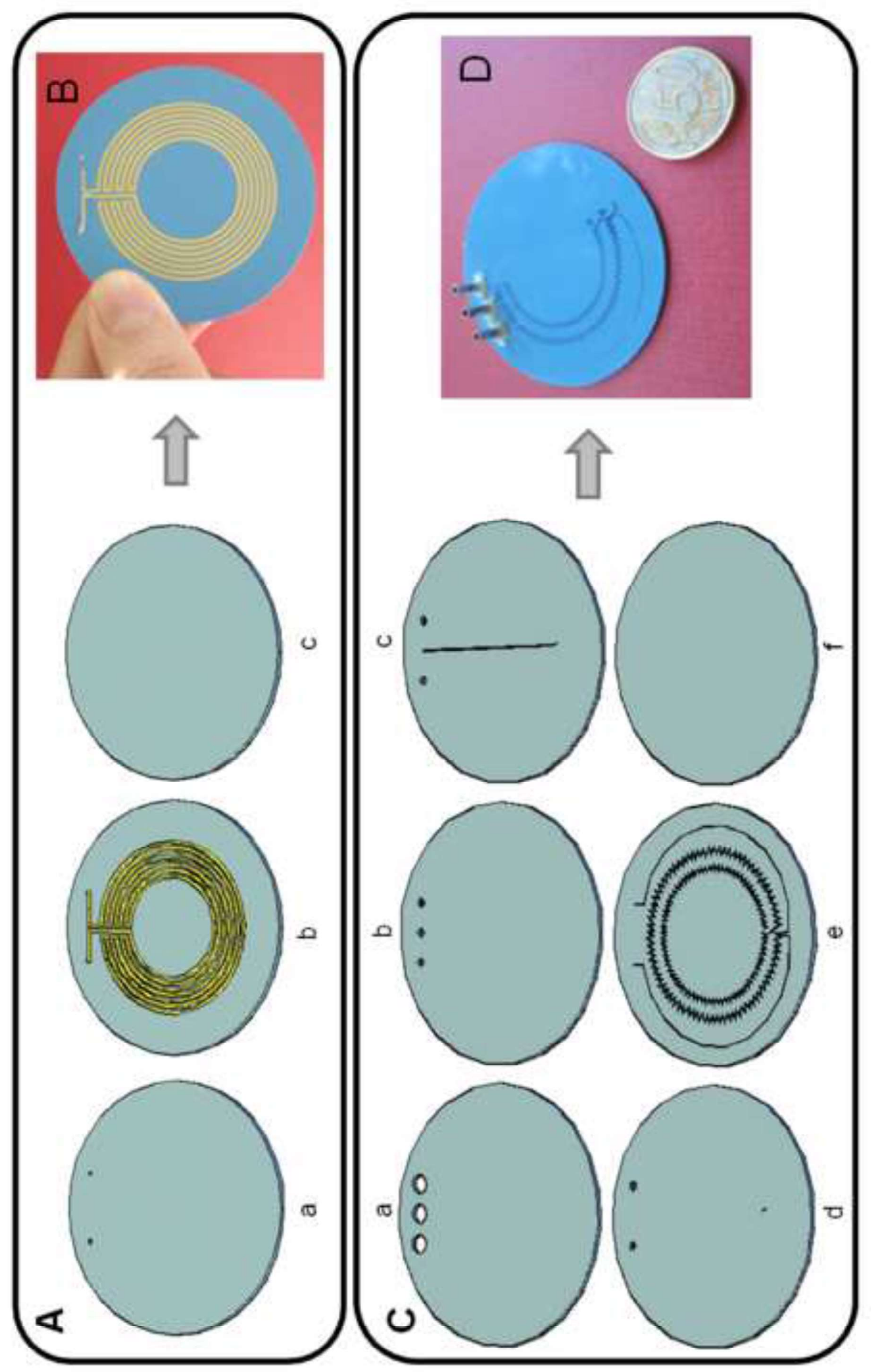




\section{LTCC MODULE}

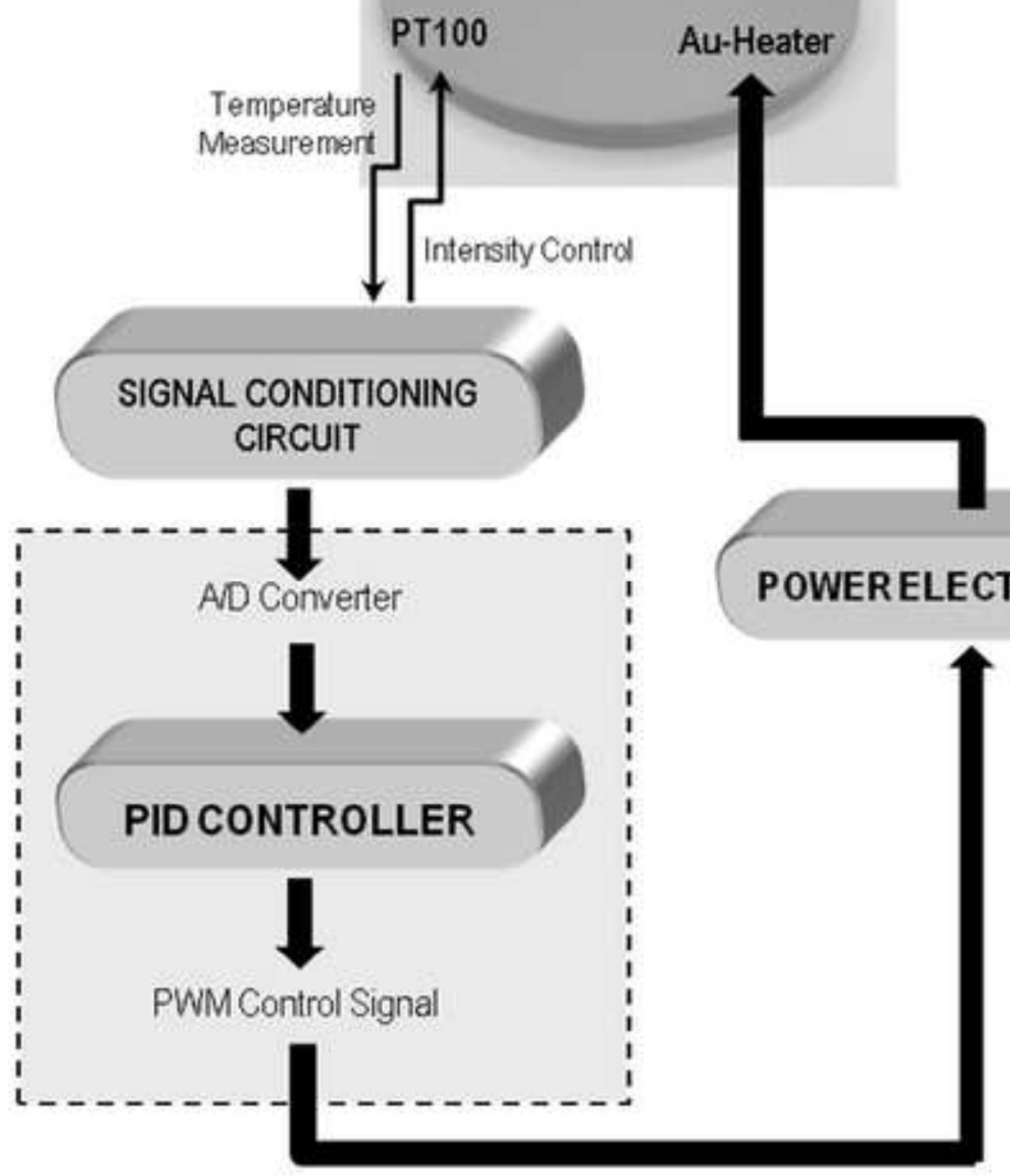



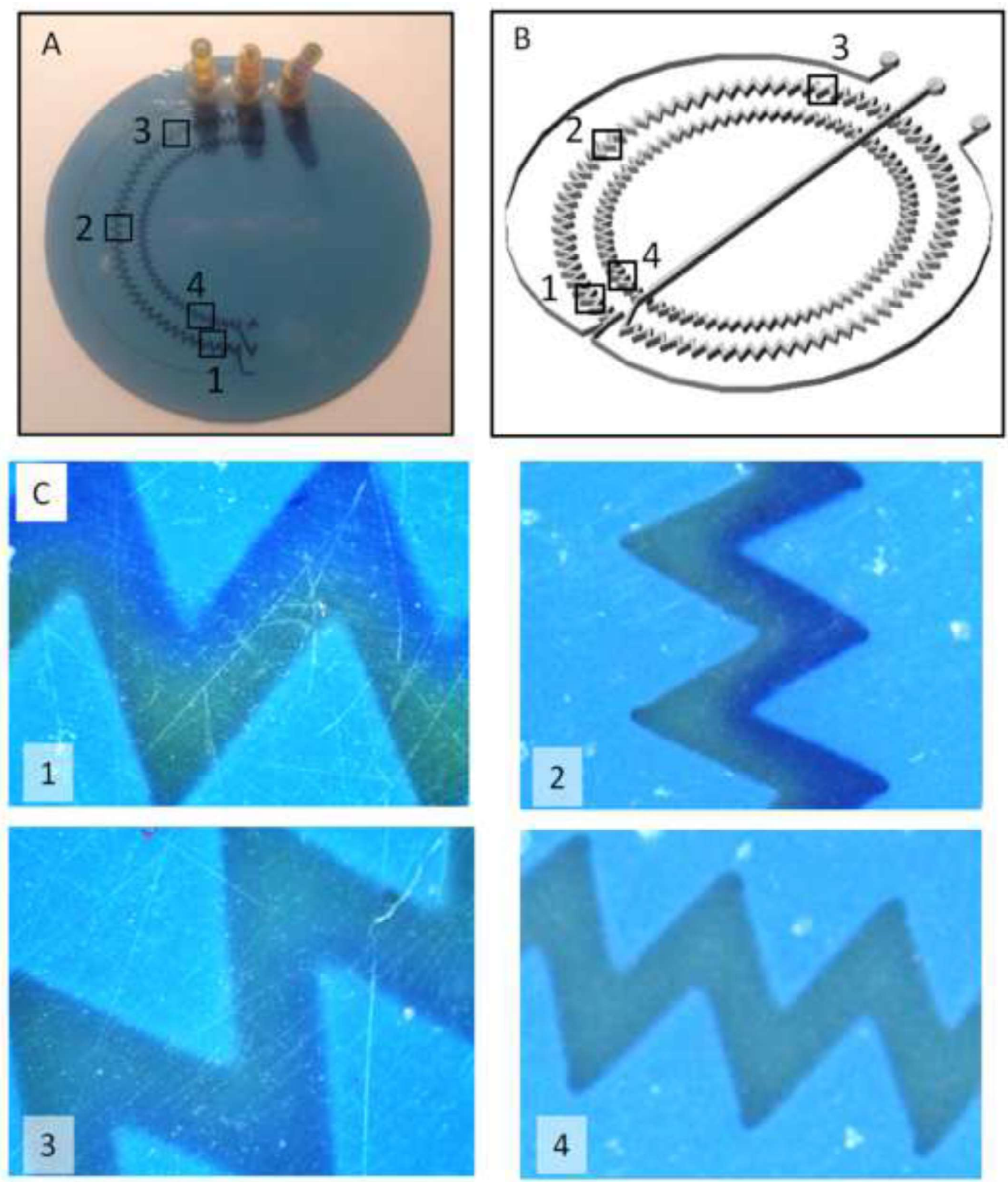

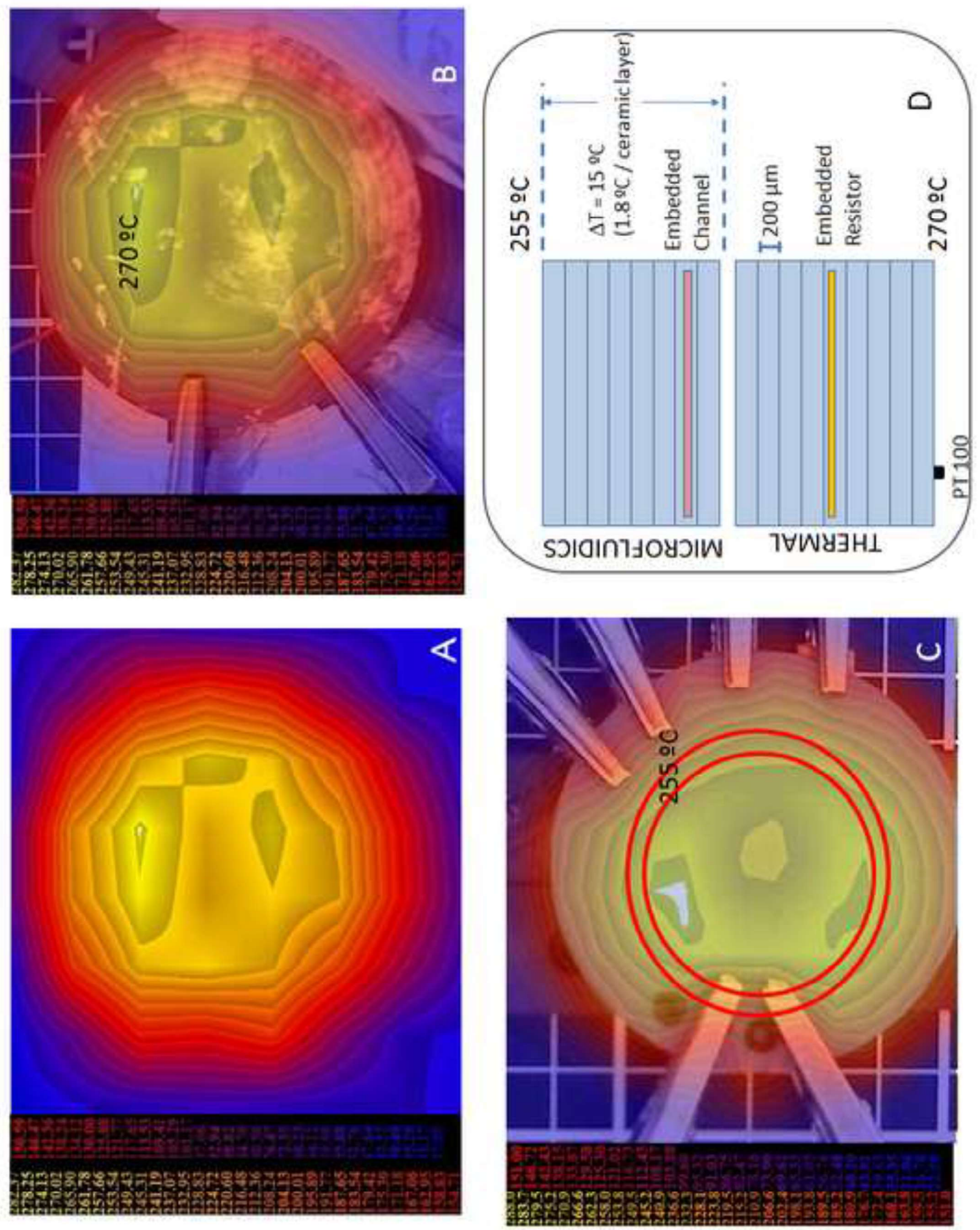

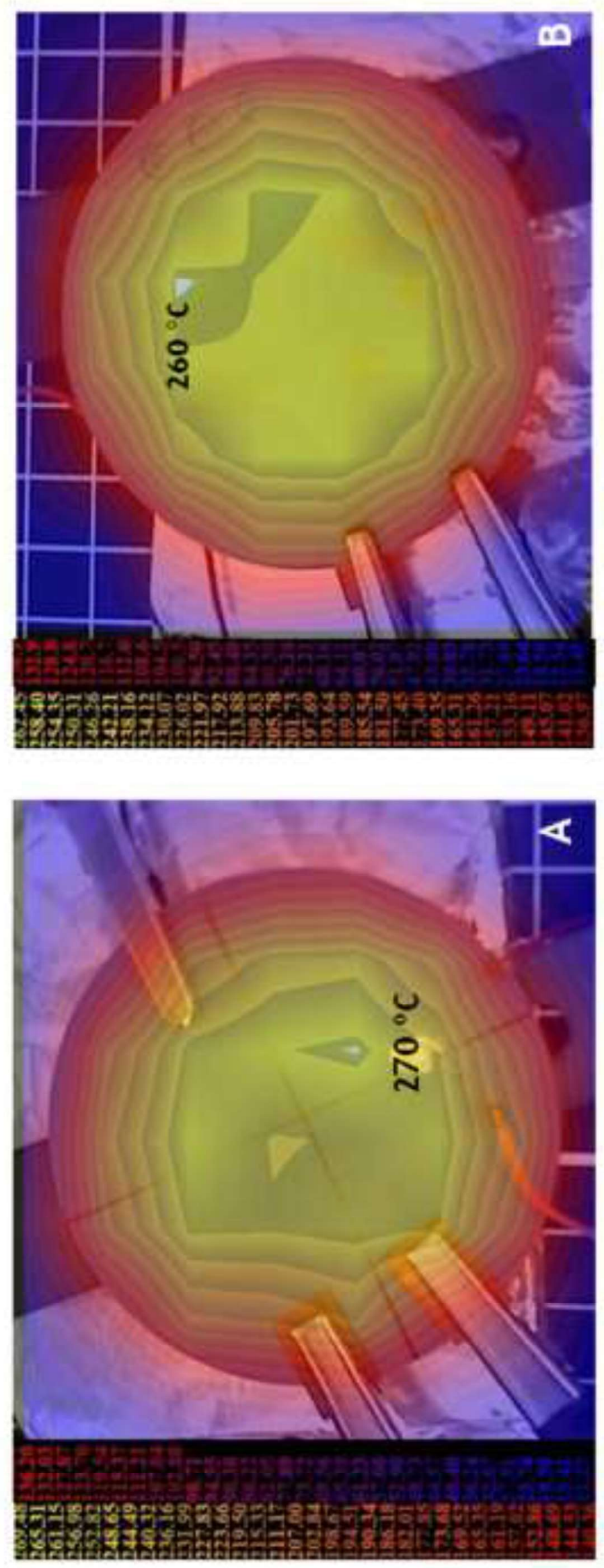

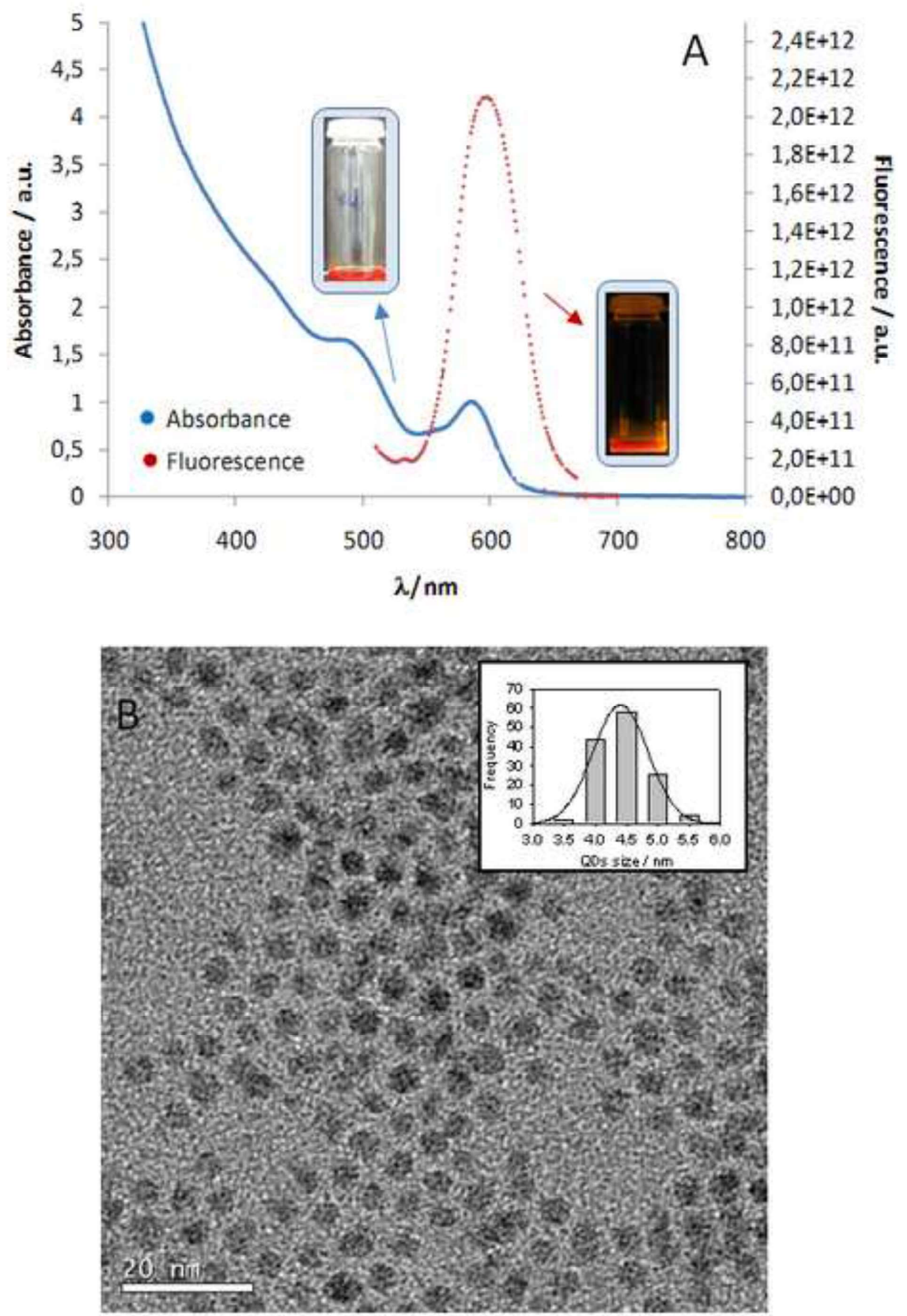